\title{
Development and the continuum of dimensional separability
}

\author{
LINDA B. SMITH \\ Indiana University, Bloomington, Indiana 47405
}

\begin{abstract}
Recent studies of adult perception may be interpreted in terms of a continuum from the integral to the separable perception of multidimensional variation. Three experiments examined the existence of such a continuum across developmental levels. These experiments are important because several developmental studies suggest that the separability of dimensions increases with age. Despite this assumed developmental trend, it was hypothesized that the relative separability of dimensions does not change with age. The results of the three experiments did not support the hypothesis. The ordering of dimensions by their relative separability changes with development. These results suggest that the relative separability and potential separability of dimensions are not fixed. In light of the results, it is hypothesized that the integrality or separability of dimensions in adult perception is primarily determined by perceptual learning and not by the inherent characteristics of combinations of stimulus dimensions.
\end{abstract}

Recent studies of adult perception may be interpreted in terms of a continuum of dimensional separability. Following an early suggestion by Shepard (1964), such a continuum has been postulated to account for the numerous empirical findings that adults' processing of multidimensional relations depends critically on the specific dimensions that vary. Garner $(1970,1974)$ and Lockhead $(1966,1972)$ have specifically suggested that some combinations of dimensions are integral whereas others are separable. Integral dimensions, such as saturation and brightness, are said to be perceived holistically. The critical relation within an integral stimulus set appears to be the overall magnitude of difference between stimuli on both integral dimensions. In contrast, separable dimensions, such as color and form, are processed in terms of the separate relations on each dimension. The critical relation within a separable stimulus set is the identity of the stimuli on each dimension. Such integral and separable dimensions may be conceptualized as the endpoints of a continuum of dimensional separability, as stimulus dimensions are not just integral or separable. Specific combinations of stimulus dimensions appear to differ in the degree to which they are processed in terms of the separate dimensional constituents.

This continuum of separability is clarified by considering two speeded classification tasks that distin-

The contribution of Melanie Lockyear in the collection of the data and the cooperation of the staff and children at the Stinesville Elementary School are gratefully appreciated. This work also benefited from comments by James Craig, David Pisoni, and Deborah Kemler. The research was funded by Grant BNS 78-13019 from the National Science Foundation. Reprint requests should be sent to the author, Department of Psychology, Indiana University, Bloomington, Indiana 47405 .
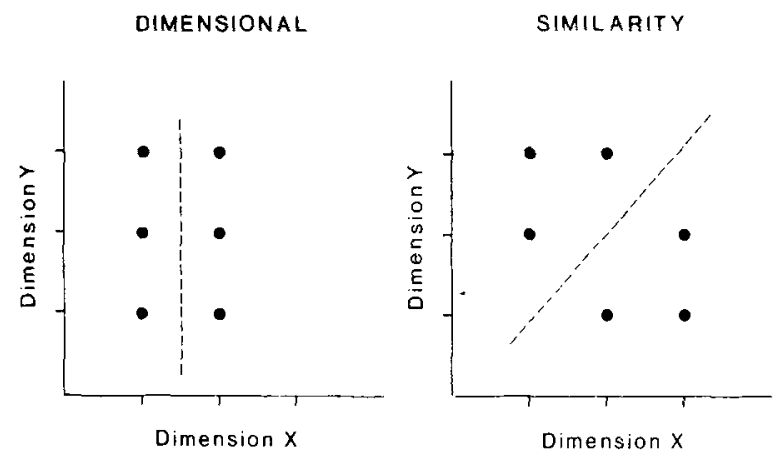

Figure 1. Sehematic representations of the two classification tasks employed in Experiment 1. Unique stimuli are represented in terms of their coordinate values on the two varying dimensions. The required classifications are indicated by dotted lines.

guish levels of separability in adult perception (Smith \& Kilroy, 1979). The structure of the two tasks is illustrated in Figure 1. The unique stimuli in each task are represented by their coordinate values on two varying dimensions, and the required classification is indicated by the dotted line. In the dimensional task, the subject is required to sort the stimuli by value on one relevant dimension. In the similarity task, however, correct classification requires attention to both varying dimensions. Processing of values on only one or the other leads to response uncertainty for four of the six stimuli. Thus, the dimensional task should be easier than the similarity task if the dimensions are processed separately. The opposite pattern is expected when the dimensions are integral, because the within-group similarity on both varying dimensions is higher and the between-group similarity is lower in the similarity task than in the dimensional task. Consistent with this analysis, Smith 
and Kilroy (1979) found that adults' sorting was faster in the dimensional task than in the similarity one when the separable dimensions of size and brightness varied, but that sorting in the similarity task was faster when the integral dimensions of saturation and brightness varied. Adults' performances with length and orientation fell between these two extremes. With this third combination of stimulus dimensions, sorting was slightly more rapid in the dimensional task than in the similarity task, but the magnitude of the difference was much smaller than it was with the separable combination of size and brightness. Thus, the three dimensional combinations can be ordered according to their relative separability, according to the degree that sorting by identity on one dimension was more rapid than sorting by the overall similarity on both dimensions.

Possible explanations of this continuum of effects of specific combinations of dimensions are complicated by developmental differences in the separability of dimensions. Specifically, in the tasks used to define dimensional integrality and separability (see Garner, 1974), young children's performances with adult-separable dimensions have been found to pattern in an adult-integral manner (Shepp, 1978; Shepp \& Swartz, 1976; Smith \& Kemler, 1977, 1978). In other words, many adult-separable dimensions appear to be integral for young children. This developmental trend has been interpreted as indicating that all dimensions are equally integral at early stages of development (e.g., Smith \& Kemler, 1978). This strong claim that differences in the processing of various stimulus dimensions emerges only with development may not be correct. If one examines young children's performances across a variety of tasksincluding those not specifically concerned with the issue of dimensional separability-it is apparent that young children can judge adult-separable dimensions such as color and form or size and brightness in a differentiated manner (e.g., Cunningham \& Odom, 1978; Kemler \& Smith, 1979; Tighe \& Tighe, 1978; Ward, in press). Indeed, young children can selectively attend to values on one adult-separable dimension more easily than adults can selectively attend to values on one integral dimension (Kemler \& Smith, 1979; Smith \& Kemler, 1978). Thus, adult-separable dimensions do not appear to be as "integral" for young children as integral dimensions are for adults. Perhaps, then, stimulus dimensions differ in their perceptual separability throughout development.

This possibility, that dimensional combinations differ in their inherent separability at all developmental levels, was the focus of the present study. This investigation is important because the claim that the separability of dimensions increases with age need not imply that all dimensional combinations are perceived equally holistically by young children. Following Garner (1974), dimensional combinations may differ in their inherent separability-across developmental levels. Although many dimensional combinations may be more difficult to separate for young children than for adults, the dimensions that are most separable for adults may also be the ones that are most separable for young children. The hypothesis, then, is that the relative separability of dimensions does not change with age. The ordering of dimensions from most integral to most separable is hypothesized to be the same at all developmental levels.

This hypothesis was investigated in the first experiment by examining older and younger children's speeded classifications in dimensional and similarity tasks structured like those shown in Figure 1. The relative separability of three dimensional combinations was examined: (1) saturation and brightnessintegral for adults (e.g., Smith \& Kilroy, 1979); (2) length and orientation-of intermediate separability for adults (Smith \& Kilroy, 1979); and (3) color and form-separable for adults and older children but not for younger children (e.g., Garner, 1974; Shepp \& Swartz, 1976). It was expected that these dimensions would be ordered from most integral to most separable in the same way for both younger and older children. Given the developmental trend of increasing separability, it was also expected that the "absolute level of separability" of the dimensional combinations would be greater for older than for younger children.

\section{EXPERIMENT 1}

\section{Method}

Subjects. Twenty-four kindergarteners (mean age: 5 years 7 months; range: $5-2$ to $6-9$ ) and 24 fourth graders (mean age: 10 years 6 months; range: $9-11$ to 11-9), attending a rural Indiana elementary school, served as subjects.

Stimuli. The stimulus values on each dimension were chosen from pilot studies such that all differences on each dimension were discriminable for the children.

The saturation-and-brightness stimuli consisted of Munsell chips of constant hue (10R) that varied in chroma (saturation) and value (brightness). The three levels of chroma were 3, 5, and 7 and the three levels of value were 2,6 , and 10 . There were two unique dimensional tasks that differed according to whether chroma or value was relevant for grouping the objects (i.e., whether dimension $X$ in Figure 1 was chroma or value). When chroma was relevant, its levels were 3 and 5; when value was relevant, its levels were 2 and 6 . All three levels on the two dimensions were employed in the two unique similarity tasks; for one of these tasks, dimension $\mathrm{X}$, as shown in Figure 1, was chroma, and for the other dimension, $\mathrm{X}$ was value.

The length-and-orientation stimuli consisted of arrows of three lengths $-1,1.25$, and $1.50 \mathrm{in}$.- and three orientations-40,60, and 80 deg counterclockwise from horizontal. In the dimensional task in which length was relevant, its levels were 1 and 1.25 in., and in the task in which orientation was relevant, its levels were 40 and $60 \mathrm{deg}$. The two unique similarity tasks were constructed as above.

In the color-form condition, the colors were an orange-yellow, orange, and a reddish-orange (Coloraid notation: YO, O, RO). The forms were isosceles triangles of equal area (1 sq in.) that 
varied in height (and thus also in base width). The three levels of height were $.75,1.00$, and 1.40 in. Phenomenally, the triangles vary in shape from "short-fat" ones to "tall-skinny" ones. In the dimensional task in which color was relevant, its levels were YO and $O$. When form was relevant, its levels were .75 and 1.00 in. The two unique similarity tasks were constructed as above.

Each stimulus was mounted on a $3 \times 5$ in. white card, and these cards were assembled into decks of 24 cards for speeded classification. Each deck corresponded to one of the classifications shown in Figure 1, and each stimulus item occurred four times within the deck.

Procedure and Design. A third of the children at each grade were assigned one of the three combinations of stimulus dimensionssaturation-brightness, length-orientation, or color-form. All children performed the similarity task in one session and the dimensional task in another. The two sessions were separated by approximately 7 days. The order in which the two tasks were performed and the assignment of the specific similarity and dimensional stimulus sets were counterbalanced within grade level for each combination of stimulus dimensions. The procedure in the two sessions was identical and as follows.

Pretraining. Prior to speeded classification, each child was taught the appropriate classification in a pretraining task. This training was deemed necessary because of the likely difficulty of some of the tasks (e.g., the dimensional task with saturation and brightness). In the pretraining task, the child was shown the six unique stimuli comprising a task divided into the two appropriate groups of three stimuli each. The child was told that the three stimuli in one group (e.g., on the right) belonged together and that the three stimuli in the second group (e.g., on the left) also belonged together. Four of the six stimuli were then haphazardly removed and the child was required to put them back into their proper groups. All mistakes were corrected. This phase of pretraining continued until the child had replaced the four stimuli correctly on 5 consecutive trials for a maximum of 12 trials. The second phase of pretraining was then begun for all children. In this phase, all six of the exemplars were removed and shuffled. The child was required to sort the exemplars correctly three consecutive times or for a maximum of eight times. All errors were recorded.

Speeded sorting. After completing the pretraining, the deck of 24 cards was shown to the child and he was told, "Now we are going to do the same thing with a whole lot of pictures but we're going to do it really, really fast." A rapid speeded sort was then demonstrated by the experimenter. The child then speed-sorted the deck five times. The decks were shuffled after each sort and the child was congratulated on his speed and encouraged to sort "even faster." Sorting times were measured via an electronic stopwatch accurate to $.10 \mathrm{sec}$. Errors during speeded sorting were recorded. Each session lasted 20-45 min.

\section{Results and Discussion}

Very few errors occurred in pretraining. The pattern of errors that did occur was nearly identical to that observed in speeded sorting. Therefore, errors in pretraining will not be specifically discussed. Preliminary analyses were also performed on speeded classification performance for each combination of stimulus dimensions separately. These analyses indicated that the pattern of performances with each combination of stimulus dimensions did not depend on which dimension in the combination served as dimension X (see Figure 1). For example, the relative difficulty (as measured by errors or sorting times) of the similarity task over the dimensional one with length and orientation did not interact with the factor of which of the two dimensions was relevant in the dimensional task [in all cases, $F(1,14)$ $\leqslant 1.10]$.

Errors in speeded classification. Errors in speeded sorting varied as a function of stimulus dimensions, task, and grade. To analyze these errors, each child's total errors on his first two and last two speeded sorts in each task were calculated. A total of 48 cards were sorted across two trials, and thus chance performance is equal to 24 errors. These initial and final error scores were submitted to analysis of variance for a 2 (grade) by 3 (stimulus dimensions) by 2 (task) by 2 (sorts-initial vs. final) mixed design. The only reliable main effects were of grade $[F(1,42)=13.32$, $\mathrm{p}<.001]$ and sorts $[\mathrm{F}(1,42)=10.80, \mathrm{p}<.001]$. Not surprisingly, older children made many fewer errors than younger children, and errors decreased from initial to final sorts. No factor interacted with that of sorts, and therefore Figure 2 shows the mean errors collapsed over initial and final sorts. Reliable interactions did emerge between stimulus dimensions and task $[F(2,42)=21.36, p<.001]$ and between stimulus dimensions, task, and grade $[F(2,42)=5.56, p<.01]$. Inspection of Figure 2 and post hoc analyses (Tukey $a=.05$ ) suggest that the interaction between stimulus dimensions, task, and grade is due to several sources.

Consider, first, performances with saturation and brightness. Both younger and older children speedsorted these stimuli with reliably fewer errors in the similarity task than in the dimensional one. This result is consistent with the view that saturation and brightness are quite integral and, thus, processed holistically across developmental levels. The difference between younger children's performances in the two tasks is reliably greater than that of older children. While this interaction is consistent with the claim that attention to differentiated dimensions in-

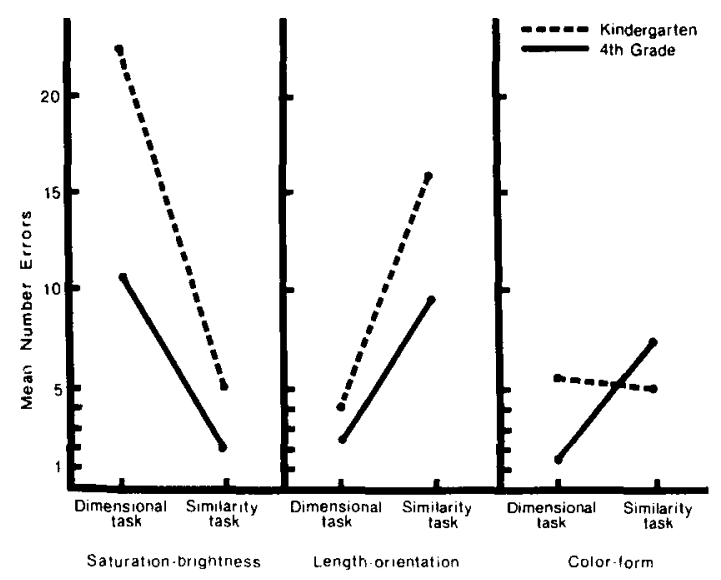

Figure 2. Mean numbers of errors committed by kindergarten and fourth grade children in the dimensional and similarity tasks employed in Experiment 1. Mean errors are shown for each of the three combinations of stimulus dimensions examined. Chance equals 24 errors. 
creases with age, it also may simply reflect the "truism" that tasks that are difficult for older children are even more difficult for younger children.

The pattern of errors with the second combination, length and orientation, were quite the opposite of those with saturation and brightness. Clearly, there are effects of the specific combinations of stimulus dimensions on performance even early in development. With length and orientation, both younger and older children made reliably fewer errors in the dimensional task than in the similarity one. Clearly, both younger and older children can separate length and orientation, and both find a task requiring selective attention to one of these dimensions easier than a classification task requiring attention to values on both dimensions. The magnitude of the effect again appears to be greater for younger than for older children. However, as suggested in the case of saturation and brightness, this interaction may simply reflect differential effects of task difficulty at the two ages.

Finally, younger and older children's performances did differ when the varying dimensions were color and form. Younger children's errors in the similarity and dimensional tasks did not differ reliably with this combination, whereas older children made reliably fewer errors in the dimensional task than in the similarity one. Apparently, older children are better able to separate and selectively attend to one dimension when the dimensions are color and form than are younger children.

The critical issue is what these patterns of errors imply about the relative separability of three dimensional combinations at the two age levels. For fourth graders, saturation and brightness are clearly the least separable dimensions. Contrary to expectations, however, marked differences between the relative separability of color and form and of length and orientation were not apparent in the group performances of the older children. With both combinations, fewer errors were made when sorting in the dimensional than when sorting in the similarity task, and the magnitude of the difference in performances in these two tasks did not differ reliably between these two conditions of stimulus dimensions [ $t(15)$ $=.98$ ]. However, an examination of individual fourth graders' performances does provide some support for the claim that color and form are more separable than length and orientation at this age level. All of the eight fourth graders who sorted in the color-form tasks made fewer errors in the dimensional than in the similarity task, but only five of the eight fourth graders who sorted in the length-orientation tasks found the similarity task more difficult. Thus, for fourth graders, saturation and brightness are the least separable dimensions, and there is weak evidence that color and form are the most separable. The ordering of the dimensions by their relative separability for kindergarteners is more surprising. As is evident in Figure 1, saturation and brightness were the least separable dimensions and length and orientation were the most separable. It is only with length and orientation that performance in the dimensional task is superior to that in the similarity task. This reversal of the expected relative separability of the combinations of color and form and of length and orientation for kindergarteners is also apparent by the measure of sorting times in the two tasks.

Sorting times. Because of the differences in error rates in speeded sorting, each child's speed scores in the two tasks were calculated by taking the mean of his two fastest sorts with four or fewer errors. The means of these speed scores are illustrated in Figure 3. Analyses of these results revealed a pattern of performances that is virtually identical to the pattern of errors in speeded sorting. Not surprisingly, a reliable main effect of grade $[F(1,42)=9.55, p<.001]$ emerged; the older children sorted much more rapidly than the younger children. Reliable interactions between task and grade $[F(1,42)=5.56, p<.05]$ and between task and stimulus dimensions $[F(2,42)=10.76$, $\mathrm{p}<.001$ ] also emerged. Post hoc analyses (Tukey $\alpha=.05$ ) revealed that younger and older children's patterns of performance did not differ when the stimulus dimensions were saturation and brightness or when the dimensions were length and orientation. The particular pattern, however, did depend on the combination of dimensions. Both younger and older children sorted the saturation-brightness stimuli more rapidly in the similarity than in the dimensional task. In contrast, both older and younger children sorted the length-orientation stimuli more quickly in the dimensional task than in the similarity task. Again, marked developmental differences in the relative separability of these two combinations were not apparent; at both age levels, length and orientation ap-

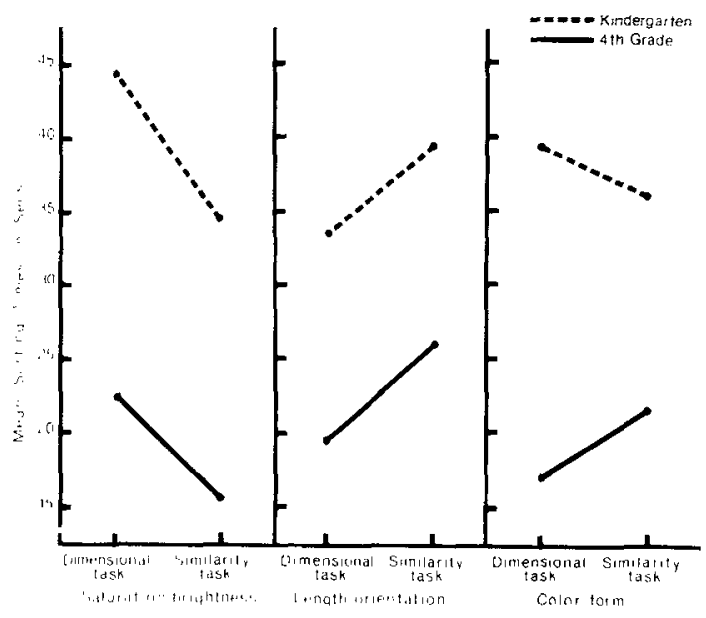

Figure 3. Mean sorting times of kindergarten and fourth grade children in the dimensional and similarity tasks employed in Experiment 1. 
pear to be more separable than saturation and brightness. However, developmental differences were again observed when the dimensions were color and form. Younger children's sorting times in the two tasks did not differ reliably with this combination, whereas older children's sorting times did. The apparently more rapid sorting by kindergarteners in the colorform similarity task than in the color-form dimensional task is due primarily to one subject. Thus, kindergarteners appear to sort color-form stimuli according to relations on two dimensions as easily as they sort these stimuli by relations on one dimension. In contrast, a clear difference between the two tasks with color and form was observed with the older children. Specifically, sorting in the dimensional task was reliably faster than was sorting in the similarity one.

These speed scores implicate the same orderings of the dimensions by their relative separability as do the error scores. For fourth graders, saturation and brightness are the least separable dimensions and marked differences in the relative separability of color-form variation and length-orientation variation were not observed. This lack of an expected difference in the relative separability of the second two combinations need not indicate that they are equally separable for fourth graders. The error scores provide a hint that color and form are the more separable of the two combinations and that such a possibly small difference may be detected by another measure of separability. The unexpected pattern of kindergarteners' performances is less easily explained. The sorting times as well as the error rates suggest a clear ordering of the dimensions by their relative separability for young children. Saturation and brightness are highly integral; color and form are somewhat integral, as performance is no better in a task organized by dimensional relations than in one organized by global relations; and length and orientation are the most separable. Contrary to the hypothesis, then, the effects of stimulus dimensions appear to interact with the developmental level of the perceiver. This interaction is also evident in the pattern of developmental differences in performances with the three combinations. The separability of saturation and brightness and of length and orientation does not change greatly from kindergarten to fourth grade. However, the separability of color and form does increase during this age span.

In light of these results, a second experiment was conducted in which a new measure of separability was employed. In this second experiment, the combinations of color-form and length-orientation were reexamined in order to clarify their relative separability for older children and to replicate the unexpected ordering of these two combinations according to their separability for the younger children.

\section{EXPERIMENT 2}

The two speeded-classification tasks used in this second experiment have also been shown to be diagnostic of differences in the separability of dimensions in both the adult literature (see Garner, 1974) and in the developmental literature (see Shepp, 1978). In both tasks, the subjects are required to speed-sort stimuli by value on one relevant dimension. In the control task, only the relevant dimension varies. In the experimental task, both the relevant and irrelevant dimensions vary. By Lockhead's (1972) analysis of these tasks, the dimensions need not be separated in the control task. The subject can sort the stimuli correctly simply by attending to the dimensionally undifferentiated relations of identity and difference. However, a dimensional analysis of the stimuli is beneficial in the experimental task. The subject (as in the dimensional task of Experiment 1) need only selectively process the values on one of the two varying dimensions to sort correctly. Thus, when dimensions are completely separable, performance in the control and experimental tasks does not differ (see Garner, 1974; Lockhead, 1972). Presumably, the dimensions are immediately apprehended and processed separately, and thus the addition of irrelevant variation in the experimental task imposes no additional processing demands (see Lockhead, 1972; Smith \& Kemler, 1978). However, when the dimensions are not completely separable, sorting is slower in the experimental task than in the control task. This interference effect from the addition of irrelevant variation is consistent with the notion that additional processing of the stimuli is required to separate the relevant from the irrelevant variation in the experimental task. According to Lockhead's analysis (1972), the subject must first separate the two dimensions before he can selectively attend to the values on the relevant dimension. The magnitude of the interference effect thus provides a measure of the speed with which dimensions are separated and may be used as an index of the relative separability of dimensional combinations.

\section{Method}

Subjects. Sixteen kindergarteners (mean age: 5 years 9 months; range: 5-5 to 6-7) and 16 fourth graders (mean age: 10 years 1 month; range: $9-3$ to 10-8) attending the same elementary school as those who participated in the first experiment served as subjects.

Stimuli. The stimuli in the experimental task consisted of four unique stimuli resulting from all combinations of two values on the dimension relevant for classification and two values on the irrelevant dimension. When the stimulus dimensions were color and form, the two form values were (height of triangle) .75 and 1.00 in. and the color values were (Coloraid notation) YO and $\mathrm{O}$. When the stimulus dimensions were length and orientation, the length values were 1.00 and 1.25 in. and the orientation values were (counterclockwise from horizontal) 40 and $60 \mathrm{deg}$. For each condition of stimulus dimensions, there were four unique control tasks. The stimuli for these tasks consisted of two unique stimuli 
that differed only on the relevant dimension; the other dimension was held constant at one of its two values. For example, the four color-form control tasks consisted of two color-relevant tasks in which form was held constant at either .75 or 1.00 and two formrelevant tasks in which color was constant at either YO or $O$. The stimuli for each task were mounted on $3 \times 5$ in. white cards and assembled into decks of 24 cards for speeded sorting. The unique stimulus items in each task occurred equally often within the deck for speeded sorting.

Procedure and Design. Half the children at each grade level were assigned the color-form tasks and half were assigned the length-orientation tasks. Each subject performed four tasks: one control task and the experimental task according to one relevant dimension (e.g., color or length) and one control and experimental task according to value on the formerly irrelevant (but now relevant) dimension (e.g., form or orientation). The order of sorting by each dimension within a condition as well as the assignment of specific control tasks were counterbalanced across conditions and grade level.

At the beginning of the experimental session, the child was told that he was to speed-sort cards according to one dimension and was then shown the values on that dimension. The deck of stimulus cards was shown to the child and a speeded sort was demonstrated by the experimenter. The child then alternately speedsorted the control and experimental decks four times each. The decks of stimulus cards were shuffled prior to each sort. Sorting times were measured via an electronic stopwatch accurate to $.10 \mathrm{sec}$. After completion of this task, the child was told that he was to do the same thing again but that he now had to sort by the second (formerly irrelevant) dimension. The two relevant values were shown to the child and the appropriate control and experimental decks were speed-sorted by the child as described above.

A session lasted $30-45 \mathrm{~min}$. Several kindergarteners required two (20-25 $\mathrm{min})$ sessions. If two sessions were required, sorting according to one dimension was completed in the first session and sorting by the other was begun in the second session.

\section{Results and Discussion}

Errors in speeded sorting occurred less than $3 \%$ of the time both in the initial two and final two sorts in each task with each dimensional combination. Therefore, sorting errors will not be discussed further. Initial sorting scores were calculated by taking the mean of each child's sorting times in his first two sorts in the control task and the experimental task. Final sorting scores consisted of the means of the last two sorts in each task. The means of these scores as a function of grade and stimulus dimensions are illustrated in Figure 4. The scores were analyzed by separate analyses of variance for each grade and by planned comparisons $(\alpha=.05)$.

Fourth graders' performance. The older children's sorting scores were submitted to an analysis of variance for a 2 (stimulus dimensions) by $2 w \cdot 2$ (dimension relevant for sorting with stimulus dimensions) by 2 (sorts-initial vs. final) by 2 (task) mixed design. The analysis revealed only main effects indicating reliable differences between initial and final sorts $[F(1,14)=48.88, p<.001]$ and between the experimental and control tasks $[\mathrm{F}(1,14)=16.45, \mathrm{p}<.005]$. As is evident in Figure 4, final sorts were more rapid than initial ones. The planned comparisons revealed several important results. First, when the stimulus dimensions were color and form, there were no dif-

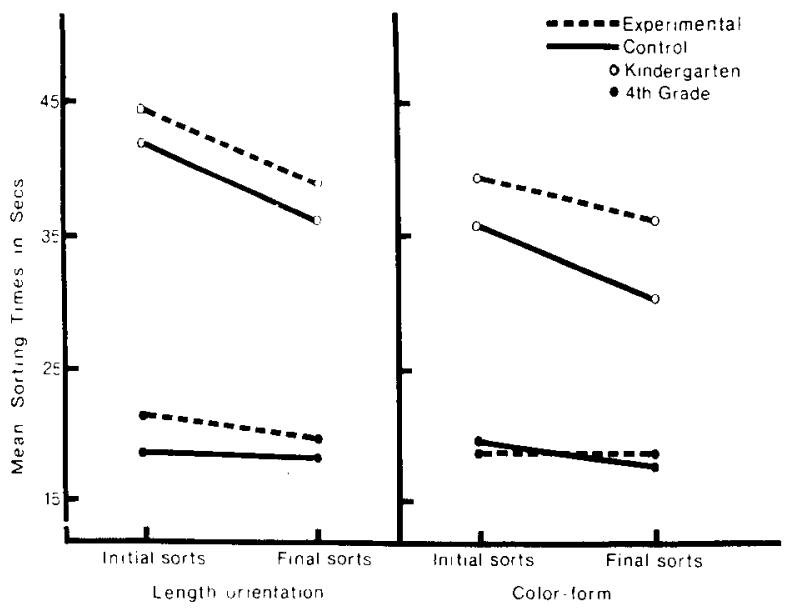

Figure 4. Mean sorting times of kindergarten and fourth grade children in the control and experimental tasks employed in Experiment 2.

ferences between the experimental and control conditions on either initial or final sorts $[t(7)<1.00$ in both cases]. This lack of an interference effect, especially on initial sorts, suggests that color and form are completely separable for these older children. In contrast, when the stimulus dimensions were length and orientation, reliable interference effects emerged both on initial sorts $[\mathrm{t}(7)=6.74, \mathrm{p}<.005]$ and on final sorts $[\mathrm{t}(7)=4.39, \mathrm{p}<.005]$. Apparently, older children do not immediately perceive length and orientation as separate dimensions of variation but must, instead, apply additional processing time to separate the two. However, the magnitude of the interference effect with length and orientation was reliably less on final than on initial sorts $[t(7)=2.07$, $\mathrm{p}<.05]$. This is an interesting result because it suggests that older children become better able to differentiate length and orientation with practice. The most critical aspect of these results is the finding that color and form are, indeed, more separable than length and orientation for fourth graders.

Kindergarteners' performance. Kindergarteners' sorting scores were also submitted to an analysis of variance for a 2 (stimulus dimensions) by $2 \mathrm{w} \cdot 2$ (dimension relevant for sorting with stimulus dimensions) by 2 (sorts-initial vs. final) by 2 (task) mixed design. The analysis revealed main effects indicating a reliable difference between initial and final sorts $[F(1,14)=29.2, p<.001]$ and between the experimental and control tasks $[F(1,14)=22.00, p<.001]$. Moreover, the interaction between tasks and stimulus dimensions was reliable $[\mathrm{F}(1,14)=5.28, \mathrm{p}<.05]$. Planned comparisons indicated reliable interference effects when length and orientation varied on both initial $[\mathrm{t}(7)=5.47, \mathrm{p}<.005]$ and final sorts $[\mathrm{t}(7)=$ $4.24, \mathrm{p}<.005$ ]. Reliable interference effects were also obtained on both initial and final sorts with color and form $[\mathrm{t}(7)=5.44, \mathrm{p}<.005$, and $\mathrm{t}(7)=6.68$, 
$p<.005$, respectively]. Furthermore, the magnitude of the interference effect did not decline between initial and final sorts with either combination [t(7) $<1.00$ in both cases]. In fact, the direction of the trend with color and form suggests an increase in the magnitude of the interference effect with practice. Apparently, younger children do not immediately perceive either combination in terms of its dimensional constituents, nor do they appear to learn to separate the dimensions more rapidly with limited practice. Again, the critical issue is the relative separability of the two combinations for these young subjects. A comparison of the magnitudes of the interference effects with the two combinations suggests that length and orientation are more separable for kindergarteners than are color and form. Specifically, the interference effect with color and form is reliably greater for kindergarteners than is that with length and orientation both on initial [ $t(14)$ $=2.86, \mathrm{p}<.01]$ and on final sorts $[\mathrm{t}(14)=5.91$, $\mathrm{p}<.005]$.

Consistent with the results of Experiment 1, the relative separability of length-orientation and colorform variation for kindergarteners appears to be opposite to that expected and opposite to that now observed with older children. Thus, the results indicate an interaction between the effects of combinations of stimulus dimensions and the age of the perceiver. The dimensions that are most separable for older children are not necessarily the ones that are most separable for younger children.

This kindergarten result was replicated in the final experiment. This replication was deemed necessary in part because of the potential importance of the findings and also because kindergarteners' sorting times in the color-form control tasks were reliably more rapid than their sorting times in the lengthorientations tasks $[\mathrm{t}(14)=3.01, \mathrm{p}<.01]$. Thus, it is possible that the larger interference effect is due to the greater discriminability (and thus, perhaps, salience) of color and form. If values on the irrelevant dimension (as well as the relevant dimension) are highly discriminable, selective attention might be more difficult not because the dimensions are difficult to separate, but because it is simply more difficult to ignore the irrelevant dimension (see, for example, Odom, 1972; Carrell, Smith, \& Pisoni, Note 1). Thus, in the next experiment, the discriminability of the lengths and orientations was increased and the interference effects for kindergarteners with color-form stimuli and length-orientation stimuli were reexamined.

\section{EXPERIMENT 3}

\section{Method}

Subjects. Twelve kindergarteners (mean age: 6 years 0 months; range: $5-7$ to 6-6), attending the same elementary school as those participating in the earlier experiments, served as subjects.

Stimuli. The color-form control and experimental tasks consisted of the same stimuli used in Experiment 2. The stimuli for the length-orientation tasks consisted of the four unique stimulus items resulting from all combinations of two length values, .75 and 1.25 in., and two orientation values (counterclockwise from horizontal), 5 and $60 \mathrm{deg}$. The sorting decks for the control and experimental tasks were arranged as in Experiment 2.

Procedure and Design. The procedure and design were the same as those of Experiment 2, with two exceptions. First, all children were assigned one control and one experimental task from each condition of dimensional combination. Half the children sorted the color-form stimuli by color and half sorted these stimuli by form. These same children also were assigned length-orientation control tasks and experimental tasks. For half the children, length was relevant; for the other half, orientation was relevant. Thus, each child sorted in four tasks, one length-orientation control task, one length-orientation experimental task, one color-form control task, and one color-form experimental task. The second difference is that sorting in all four tasks was intermixed. The resulting 20 sorts, 5 in each of the 4 tasks, were performed in a random order that was unique for each child. The experimental session lasted 30-45 $\mathrm{min}$.

\section{Results and Discussion}

Errors during speeded sorting occurred less than $2 \%$ of the time on both the initial and final sorts in each task. Initial and final sorting scores were calculated by taking the mean of each child's first two sorts in each task and the mean of his final two sorts in each task. The means of these scores for each combination of stimulus dimensions are illustrated in Figure 5 . These results were analyzed by a series of planned comparisons. First, reliable interference effects emerged both with length and orientation and with color and form. Specifically, sorting in the experimental task was slower than it was in the control task when length and orientation varied on both initial $[\mathrm{t}(11)=7.82, \mathrm{p}<.001]$ and final sorts $[\mathrm{t}(11)$ $=5.31, p<.005]$ and when color and form varied on initial $[\mathrm{t}(11)=11.65, \mathrm{p}<.001]$ and final sorts [ $\mathrm{t}(11)$ $=10.51, \mathrm{p}<.001]$. Second, the interference effect did not decrease reliably from initial to final sorts

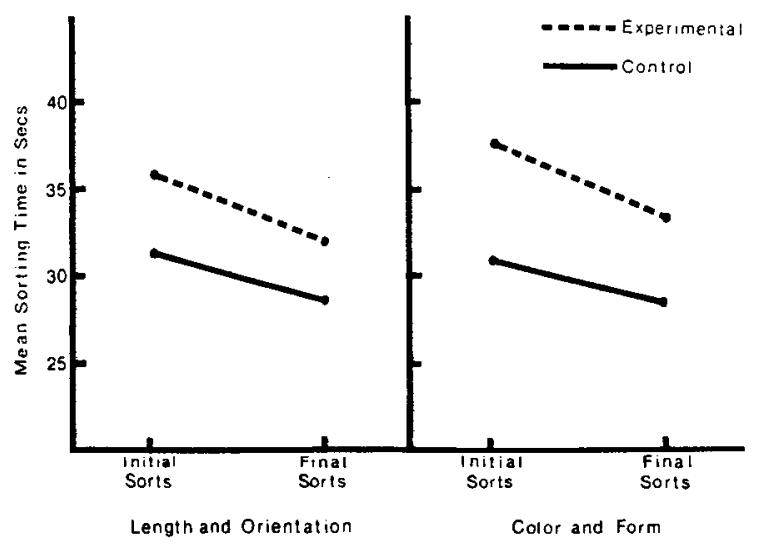

Figure 5. Mean sorting times of kindergarteners in the control and experimental tasks employed in Experiment 3. 
either for length and orientation $[\mathrm{t}(11)=1.42]$ or for color and form $[\mathrm{t}(11)<1.00]$. Again, the critical question is whether the interference effect with color and form was greater than with length and orientation. The answer is yes, both on initial sorts [t(11) $=1.92, \mathrm{p}<.05]$ and on final sorts $[\mathrm{t}(11)=2.00$, $p<.05]$. The results in this final experiment, then, bolster the surprising results from the first two experiments. Contrary to expectations, length and orientation appear to be differentiated somewhat more easily by kindergarteners than are color and form.

\section{GENERAL DISCUSSION}

The results in the three experiments do not support the hypothesis of a constant ordering of stimulus dimensions by their relative separability throughout development. On the one hand, both older and younger children's performances depended on the specific dimensions that varied. On the other hand, the differences in performances with the three combinations by younger children were not parallel to the differences observed with older children. Although saturation and brightness were clearly the least separable dimensions at both age levels, the combination that was the most separable of the three for older children was not the combination that was the most separable for younger children. This developmental reversal of the relative separability of color-form variation and length-orientation variation suggests that the continuum of separability in adult perception does not simply reflect fixed differences in the way specific stimulus dimensions are processed. Dimensions that are relatively difficult for a young child to process separately may be the very ones that are most easily processed separately by the same child when he is older. The implication is that the continuum of separability in adult perception is a product of development.

However, the developmental trend in perception is not a simple one. There is much evidence for Gibson's (1969) principle that perception becomes increasingly differentiated with development and learning. Indeed, the present findings are consistent with this principle. The separability of color and form clearly increases with age, and older children are more likely to learn to separate dimensions rapidly than are younger children. The complicating aspect of the results is the finding that the precise nature of the developmental trend depends on the specific stimulus dimensions. In the present study, marked developmental differences in the separability of saturation and brightness or length and orientation were not observed. In contrast, the evidence indicates that color and form are processed quite differently by younger and older children. Moreover, it is only this result with color and form that is consistent with previous investigations of the development of dimen- sional separability (e.g., Kemler \& Smith, 1978; Shepp \& Swartz, 1976; Smith \& Kemler, 1979). One might argue that marked developmental differences in the apprehension of saturation and brightness are not to be expected, as these dimensions are extremely integral for adults (e.g., Smith \& Kemler, 1978). The lack of developmental differences with length and orientation, however, is surprising. If there is a general trend toward increasing separability, length and orientation should be measurably less separable for younger children than for older ones. It is possible that, if even younger children were tested, we might find that the separability of length and orientation does increase dramatically with age. However, it is clear that the rate of development as well as the precise nature of the trend depends on the specific stimulus dimensions. In particular, the separability of dimensions may develop in a restricted way, for specific dimensions one at a time.

What might be the source of this developmental change and thus the continuum of stimulus effects in adult perception? Perhaps the developmental trend and the integrality-separability distinction simply reflect perceptual learning rather than anything basic or universal about the processing of multidimensional variation. Following Gibson (1969), the specific dimensions that are detected and processed selectively are those that are of value for discriminating objects in the world. We learn to detect and differentiate the particular dimensions that are relevant for distinguishing between objects. The suggestion, then, is that the separability of dimensions is not fixed but, rather, changes with specific practice at detecting specific relations. In this context, it is not at all surprising that the relative separability of dimensions is not constant across perceivers. This analysis is bolstered by several findings that performances in the tasks used to define integrality and separability depend on learning and instructions to the subjects as well as the specific dimensions that are varied (Foard, 1979; Kemler \& Smith, 1979; Smith, 1979; Ward, in press).

Still, there may be "inherent" differences between stimulus dimensions in the ease of learning to process them separately. The extreme integrality of saturation and brightness across developmental levels is consistent with the notion that some combinations of stimulus dimensions pose impassable boundaries to complete separability. Moreover, the particular interaction observed between stimulus effects and development is not readily explained by specific learning about specific dimensions. It is difficult to imagine that 5-and 6-year-olds have had more experience discriminating lengths and orientations than they have had discriminating colors and forms. In fact, there is considerable evidence that some orientation discriminations are quite difficult for young children (e.g., Ghent, 1961; Gibson, Gibson, Pick, \& Osser, 
1962). However, in another sense, color and form may be more "difficult" dimensions than length and orientation. Both color and form are highly complex dimensions that are not well understood and highly studied by students of perception. Both dimensions are built up of "subdimensions" or "features" in a way that is not easy to specify. Thus, color and form may be "higher order" dimensions that appear simple only to the mature perceiver. The intrinsic complexity of these dimensions may be apparent only in the developmental trend. Thus, there may be inherent differences between combinations of stimulus dimensions. Contrary to the initial hypothesis, however, such differences are not simply reflected in adult perception.

In conclusion, the perception of multidimensional variation depends critically on two factors: the combination of stimulus dimensions and the developmental level of the perceiver. The present results indicate that these factors interact in a quite complex manner. Stimulus effects are not uniform across development, and developmental differences are not constant across stimulus dimensions. Thus, neither the relative separability nor the potential separability of dimensions appears fixed. How we perceive multidimensional relations may be determined in a complicated way by both the history and skills of the perceiver and the nature of the stimulus dimensions themselves.

\section{REFERENCE NOTE}

1. Carrell, T. D., Smith, L. B., \& Pisoni, D. B. Some perceptual dependencies in speeded classification of vowel color and pitch (Research on Speech Perception, Progress Report No. 5). Bloomington: Indiana University, 1979.

\section{REFERENCES}

Cunningham, T. G., \& Odom, R. D. The role of perceptual salience in the development of analysis and synthesis processes. Child Development, 1978, 49, 815-823.

FOARD, C. F. Determinants of perceptual analysis. Unpublished doctoral dissertation, University of Pennsylvania, 1979.

Garner, W. R. The stimulus in information processing. American Psychologist, 1970, 25, 350-358.

GARNER, W. F. The processing of information and structure. Potomac, Md: E-lbaum, 1974.
Ghent, L. Form and its orientation: A child's eye view. American Journal of Psycholcgy, 1961, 74, 177-190.

GiBson, E. J. Principles of perceptual learning and development. New York: Appleton-Century-Crofts, 1969.

Gibson, E. J., Gibson, J. J., Pick, A. D., \& Osser, H. A developmental study of the discrimination of letter-like forms. Journal of Comparative and Physiological Psychology, 1962, 55, 896-906.

Kemler, D. G., \& Smith, L. B. Is there a developmental trend from integrality to separability in perception? Journal of Experimental Child Psychology, 1978, 26, 498-507.

Kemler, D. G., \& Smith, L. B. Accessing similarity and dimensional relations: Effects of integrality and separability on the discovery of complex concepts. Journal of Experimental Psy. chology: General, 1979, 108, 133-150.

LOскнEAD, G. R. Effects of dimensional redundancy on visual discrimination. Journal of Experimental Psychology, 1966, 72, 95-104.

Lockhead, G. R. Processing dimensional stimuli: A note. Psychological Review, 1972, 79, 410-419.

ODом, R. D. Effects of perceptual salience on the recall of relevant and incidental dimensional values: A developmental study. Jouinal of Experimental Psychology, 1972, 92, 285-291.

SHFPARD, R. N. Attention and the metric structure of the stimulus space. Journal of Mathematical Psychology, 1964, 1, 54-87.

Shepp, B. From perceived similarity to dimensional structure: in new hypothesis about perceptual development. In E. Rosch \& E. B. Lloyt (Eds.), On the nature and principle of formation of categories. Hillsdale, N.J: Erlbaum, 1978.

Shepp, B. E., \& Swartz, K. B. Selective attention and the processing of iniegral and noniztegral dimensions: A developmental study. Journal of Experimental Child Psychology, 1976, 22, 73-85.

Sмiтн, i. B. Perceptual development and category generalization. Chila Development, 1979, 50, 705-715.

Smith, L. B., \& Kemler, D. G. Developmental trends in free classification: Evidence for a new conceptualization of perceptual development. Journal of Experimental Child Psychology, $1977,24,279-298$.

Smith, L. B., \& Kemler, D. G. Levels of experienced dimensionality in children and adults. Cognitive Psychology, 1978, 10, $502-532$.

Smith, L. B., \& Kilroy, M. C. A continuum of dimensional separability. Perception \& Psychophysics, 1979, 25, 285-291.

Tighe, T. J., \& Tighe, L. S. A perceptual view of conceptual development. In R. D. Walk \& H. L. Pick (Eds.), Perception and experience. New York: Plenum Press, 1978.

W $_{\text {ARD, }}$ T. B. Separable and integral responding by children and adults to the dimensions of length and density. Child Development, 1980 , in press.

(Received for publication March 3, 1980; revision accepted April 16, 1980.) 